**Accepted and in press at Journal of Experimental Child Psychology (Feb, 2022)

\title{
Neural Correlates of Familiar and Unfamiliar Action in Infancy
}

\author{
Haerin Chung ${ }^{1}$, Marlene Meyer ${ }^{1,2}$, Ranjan Debnath ${ }^{3,4}$, Nathan A. Fox ${ }^{4}$ \& Amanda Woodward ${ }^{1}$ \\ ${ }^{1}$ University of Chicago, Chicago, USA \\ ${ }^{2}$ Donders Institute, Radboud University, Nijmegen, the Netherlands \\ ${ }^{3}$ Leibniz Institute for Neurobiology, Magdeburg, Germany \\ ${ }^{4}$ University of Maryland, College Park, USA
}

\begin{abstract}
Author Contributions
Haerin Chung: Conceptualization, Investigation, Methodology, Visualization, Writing - Original Draft; Marlene Meyer: Conceptualization, Methodology, Writing - Review \& Editing; Ranjan Debnath: Methodology, Software, Writing - Review \& Editing; Nathan A. Fox: Writing - Review \& Editing, Supervision, Funding acquisition; Amanda Woodward: Conceptualization, Writing Review \& Editing, Supervision, Funding acquisition
\end{abstract}

\footnotetext{
${ }^{*}$ Corresponding Author

haerinchung@uchicago.edu

Department of Psychology

The University of Chicago

5848 S University Ave, Chicago, IL, 60637
} 
Neural Correlates of Familiar and Unfamiliar Action in Infancy

\section{Abstract}

Behavioral evidence shows that experience with an action shapes action perception. Neural mirroring has been suggested as a mechanism underlying this behavioral phenomenon. Suppression of EEG power in the mu frequency band, an index of motor activation, typically reflects neural mirroring. However, contradictory findings exist regarding the association between mu suppression and motor familiarity in infant EEG studies.

In this study, we investigated the neural underpinnings reflecting the role of familiarity on action perception. We measured neural processing of familiar (grasp) and novel (tool-use) actions in 9-and-12-month-old infants. Specifically, we measured infants' distinct motor/visual activity and explored functional connectivity associated with these processes. Mu suppression was stronger for grasping than tool-use, while significant mu and occipital alpha (indexing visual activity) suppression were evident for both actions. Interestingly, selective motor-visual functional connectivity was found during observation of familiar action, a pattern not observed for novel action. Thus, the neural correlates of perception of familiar actions may be best understood in terms of a functional neural network, rather than isolated regional activity.

Our findings provide novel insights on analytic approaches for identifying motor-specific neural activity while also considering neural networks involved in observing motorically familiar versus actions.

\section{Keywords}

Action perception, Infants, Mu rhythm, Functional connectivity, Motor familiarity 
Neural Correlates of Familiar and Unfamiliar Action in Infancy

There is a close connection between action production and action perception that emerges early in development. Behavioral studies have demonstrated that as infants gain experience with new actions, there are also changes in their anticipation and understanding of other's actions (Ambrosini et al, 2013; Filippi \& Woodward, 2016; Gerson \& Woodward, 2014; Kanakogi \& Itakura, 2011; Krogh-Jespersen et al., 2018,2020; Libertus \& Needham, 2010; Sommerville et al., 2005, 2008). Neural mirroring, the recruitment of similar neural activity during the production and perception of action, has been suggested as a mechanism that links action experience and action perception (Gallese et al., 1996; Hunnius \& Bekkering, 2014; Marshall \& Meltzoff, 2014; Pineda, 2005; Woodward \& Gerson, 2014). Research into this possibility has made use of the EEG mu rhythm, which is oscillations in the alpha band (adults $8-13 \mathrm{~Hz}$, infants $6-9 \mathrm{~Hz}$ ) recorded over the sensorimotor cortex, as a potential index of neural mirroring (Cochin et al., 1999; Fox et al., 2016; Lepage \& Théoret, 2006; Muthukumaraswamy \& Johnson, 2004). The mu rhythm is found to be suppressed in power (also called mu suppression) during both action execution and action observation, and cortical source localization studies provide supportive evidence that the mu rhythm is associated with activity in the sensorimotor cortex in adults (Hari et al., 1997; Toro et al., 1994). However, deploying measures of the mu rhythm in infant EEG studies has led to seemingly contradictory findings on the unique role of one's motor expertise on the level of mu suppression. That is, research with infants has demonstrated an association between infants' motor experience and the level of mu power suppression during action observation. Yet, other studies have revealed similar patterns in mu suppression not only for motorically familiar actions such as grasping actions, but also for actions that are completely outside of infants' motor repertoire such as tool-use actions. This calls into question whether the mu rhythm is modulated by the motoric familiarity of an action or not. In the current study, we aimed to investigate the neural underpinnings of observing familiar and novel actions within the same sample of infants and to identify the neural processes that are uniquely associated with observing motorically familiar actions.

A growing body of studies indicates that mu suppression is associated with and affected by infants' experience producing actions (Cannon et al., 2016; Gerson, Bekkering et al., 2015; Paulus et al., 2012; van Elk et al., 2008; Yoo et al., 2016). For instance, Cannon et al. (2016) 
found that 9-month-old infants who were more proficient graspers showed stronger mu suppression when observing others' grasping actions. In an experimental intervention, Gerson, Bekkering et al., (2015) reported that active experience with a novel action, but not passive observational experience, engendered changes in mu power when 10-month-old infants subsequently heard the sound associated with that action. Moreover, Filippi et al. (2016) found that 7-month-old infants' likelihood of imitating the goals of others' action was correlated with variations in mu suppression during their own reaching and during observation of others' reaching actions. Together, these findings highlight the potential role of motor experience in modulating mu suppression during action observation.

However, another body of studies with infants at the same age ( 9 months) and analyzing neural activity in the same frequency range $(6-9 \mathrm{~Hz})$ recorded over the sensorimotor cortex (central sites), found mu suppression when infants observe actions and events that they have not had active experience with (de Klerk et al., 2016; Southgate \& Begus, 2013; Virji-Babul et al., 2012). In a between-subjects design, Southgate and Begus (2013) reported that 9-month-old infants showed mu suppression during anticipation of grasping as well as novel tool-use actions and even while viewing the movements of self-propelled objects. Similar findings were reported by Virji-Babul et al., (2012), in 4-to-11 month-old infants in a within-subjects design. These findings challenge the assumption that mu suppression is a unique neural correlate of the motor familiarity of observed actions.

Thus, while behavioral evidence illustrates a connection between production and perception in infants, the neural processes associated with this link are in question. Mu suppression has been a valuable candidate, extensively used as an index of neural processes associated with action production and perception. However, mu suppression as an isolated neural signal, may not unambiguously reflect motor familiarity of the observed action. One reason could be related to the potential influence of occipital (visual) alpha, which occurs in response to the presentation of a visual stimulus (Fox et al., 2016; Hobson \& Bishop, 2016). Reliable separation of mu from occipital alpha is critical for adequate interpretation of visual and motor effects on action perception (Hobson \& Bishop, 2016). Yet, it remains a methodological challenge to parse apart 
mu from occipital alpha (Hobson \& Bishop, 2016). To resolve this issue, reporting alpha activity recorded over multiple brain regions (at least including activity over visual areas) has been recommended (Perry \& Bentin, 2010; Bowman et al., 2017; Cuevas et al., 2014; Fox et al., 2016). This is a helpful starting point, though reporting multi-region activity is not sufficient to parse apart whether mu suppression is reliably discernable from visual alpha. Others explore the relation between central and occipital alpha (Tangwiriyasakul et al., 2013) or encourage researchers to use methodological controls (e.g. using baselines that control for attentional demands) as an additional approach to distinguish action-specific processes from visual processes (Fox et al., 2016). Infant researchers have reported alpha activity recorded over multiple regions (Southgate \& Begus, 2013; Cannon, et al., 2016; Yoo et al., 2015; Filippi et al., 2016; de Klerk et al., 2016); however, it is rarely the case that additional approaches are endorsed to ensure that activity recorded over central and occipital sites are reliably independent (but see Filippi et al., 2016; de Klerk et al., 2016).

In addition, it is not only important to reliably distinguish between motor and visual processes, but also understanding how motor and visual processes are coordinated together during action perception is crucial, as visual processes may be inherent during action processing (Fox et al., 2016). Using mu suppression in isolation as a neural marker of action processing may overlook potentially important network connectivity. Understanding the dynamic inter-regional communications among networks in the brain can provide insights to the basis of infants' behavioral repertoire (Gao et al., 2017). However, the functional relation of motor and visual processes on action perception in infancy remains unclear. Debnath and colleagues (2019) demonstrated enhanced connectivity between EEG signal recorded at central and occipital scalp locations during infants' observation of a familiar grasping action. It is an open question whether this functional connectivity is also evident during the observation of novel actions. Exploring functional connectivity between mu and occipital alpha, rather than mu as an isolated neural marker, could be the key in reconciling the discrepancy across findings (Bowman et al., 2017). That is, functionally related neural systems may better index the underlying mechanism that uniquely governs perception of motorically familiar action. It could be that active 
Neural Correlates of Familiar and Unfamiliar Action in Infancy

experience in an action builds on and strengthens communication between network processes (Johnson, 2000), which may not be evident during observation of novel action.

\section{Current Study}

In the current study, we aimed to better understand the neural correlates of perceiving motorically familiar and unfamiliar actions, beyond changes in mu power. For this purpose, we collected EEG while 9-and-12-month-old infants viewed a familiar action (grasping) and a novel action (tool use) in a within-subjects design. Infants begin to produce reaches by 5 months and become highly skilled by 9 months (Bertenthal \& Clifton, 1998). The ability to engage in tool-use actions becomes robust during the second year when infants become able to spontaneously engage with novel tools (Keen, 2011). Thus at both 9 and 12 months of age, reaching is a motorically familiar action, while engagement with a novel tool is unfamiliar. Therefore, our main analyses were conducted across these two ages. We drew on several recent methodological approaches to more clearly capture distinct EEG mu measured at the central electrodes from EEG alpha measured at the occipital electrodes (see Bowman et al., 2017; Fox et al., 2016; Hobson \& Bishop, 2016). Importantly, we assessed mu suppression relative to a baseline that was visually similar to the action videos. This was done to reduce the extent to which mu activity might be confounded with occipital alpha response to visual novelty (Nyström et al., 2011). Then, offering a tighter comparison with a within-participant design, we compared whether suppression of mu / occipital alpha is modulated by motor familiarity. We also assessed infants' skills in grasping and capacity to engage in the novel tool use action so that we could evaluate correlations between motor competence and mu / occipital alpha suppression during action observation. Lastly, we explored functional connectivity between central and occipital neural activity during perception of familiar and unfamiliar action to evaluate coordination of motor and visual processes. 
Neural Correlates of Familiar and Unfamiliar Action in Infancy

\section{Method}

\section{Participants}

Forty-nine full-term 9-month-olds (21 infants; $M=9$ months 21 days, SD= 10; range: 9;0 $10 ; 15 ; 13$ males) and 12-month-old infants (28 infants; $M=12$ months 21 days, $S D=17.8$; range: $11 ; 15-13 ; 15 ; 11$ males) participated in this study ${ }^{1}$. Participants were recruited in an urban Midwest region in the United States. Infants represented a diverse racial background (52\% European, 15\% Hispanic, 16\% African-American, 5\% Asian, and 12\% mixed-racial) and were from relatively highly educated families (91\% caregivers reported receiving bachelor's or higher degrees). Thirteen infants were excluded from analyses due to being distressed shortly after application of the EEG electrode net (2), unusable EEG data prior to data analysis (6), and for not having at least three artifact-free EEG trials per condition after all EEG processing steps (5). The final dataset consists of 36 infants (17 9-month-olds, 19 12-month-olds). This study was approved by the campus Institutional Review Board at the University of Chicago.

\section{Procedure}

Upon arrival in the laboratory, infants' caregivers were first informed about the testing procedure by Experimenter 1 (E1) and filled out a written consent form. During this process, Experimenter 2 (E2) measured the infants' head circumference and prepared the best-fit net in the EEG testing room. Then, the infants and caregivers were accompanied to the EEG testing room. The infants sat in an infant-friendly high-chair or on the caregiver's lap and were fitted with a 128-sensor HydroCel Geodesic Sensor Net (Electrical Geodesics, Inc., Eugene, OR). Impedances were kept below $100 \mathrm{k} \Omega$ where possible. The EEG was recorded at $1000 \mathrm{~Hz}$ (Net Station software, EGI, v4.5.7) and Cz electrode was used as an online reference. Once infants wore the EEG net, E1 took pictures and a video to document individual net placement, and these pictures were sent to caregivers after their visit. Infants faced a computer screen on a

\footnotetext{
${ }^{1}$ We based the sample size on the studies that have utilized similar experimental designs (Cannon et al., 2016 analyzed 21 participants, and Southgate \& Begus, 2013 analyzed 33 participants with 11 in each condition).
} 
Neural Correlates of Familiar and Unfamiliar Action in Infancy

table at approximately $70 \mathrm{~cm}$ distance on which stimuli videos were presented via Eprime 2.0 (Schneider et al., 2012). When videos were presented via Eprime, triggers indicating the onset and end of videos were simultaneously sent to the EEG recording software Net Station. See Figure 1 for room set up. E1 sat behind a screen throughout the video presentation hiding from the infants' view and monitored the infant during the experimental procedure. Parents were asked to avoid interacting with their infants as much as possible, except when the infants were in distress. E2 was in the next room monitoring the recordings of EEG and videos of the session. Videos of the sessions were recorded from two different camera views. One camera was placed in front of the infant to capture a close-up view of the infant, and another camera was placed behind the infant to record the computer monitor presenting the video stimulus during observation. These recordings in the experimental session were video-coded offline so that it can be time-locked to the EEG recording. The EEG session consisted of an action observation and an action execution phase. 
Neural Correlates of Familiar and Unfamiliar Action in Infancy

Observation phase

A. Schematic time-line of Observation trial (a)Grasp Condition

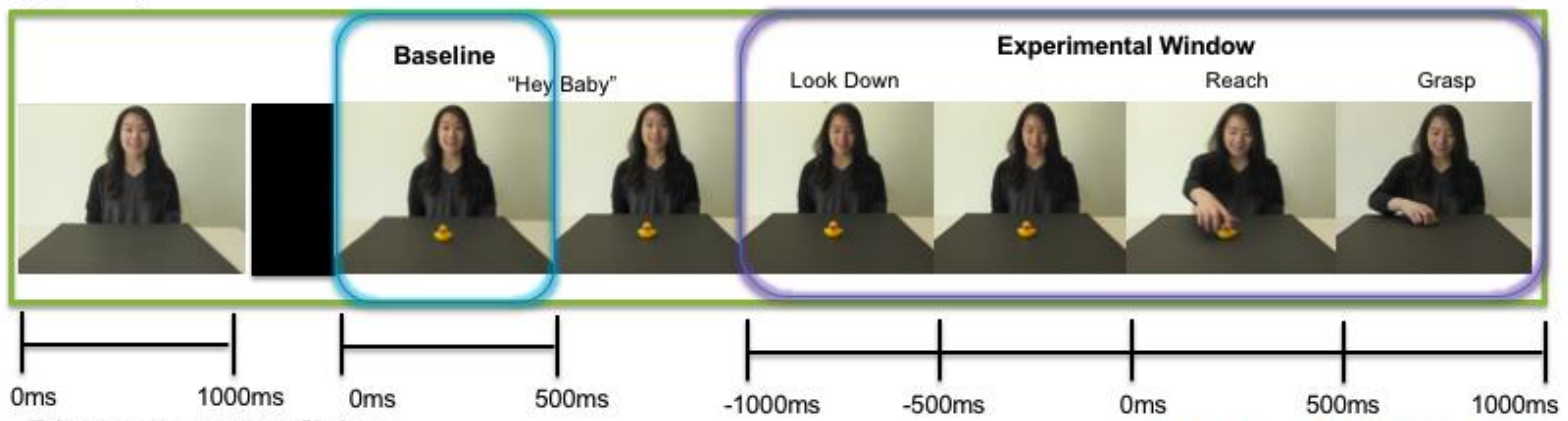

(b)Cane-use Condition

*0- time locked to movement onset

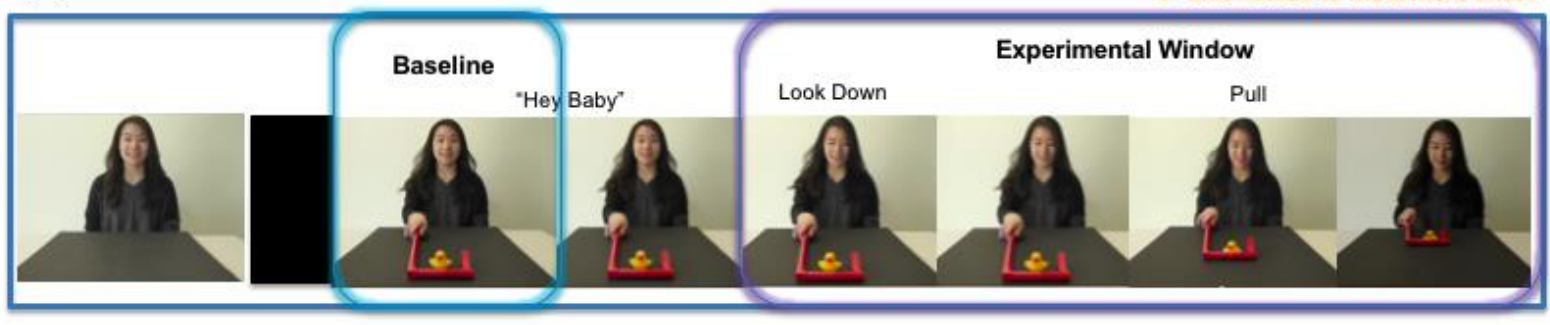

B. Experimental set up during Observation phase
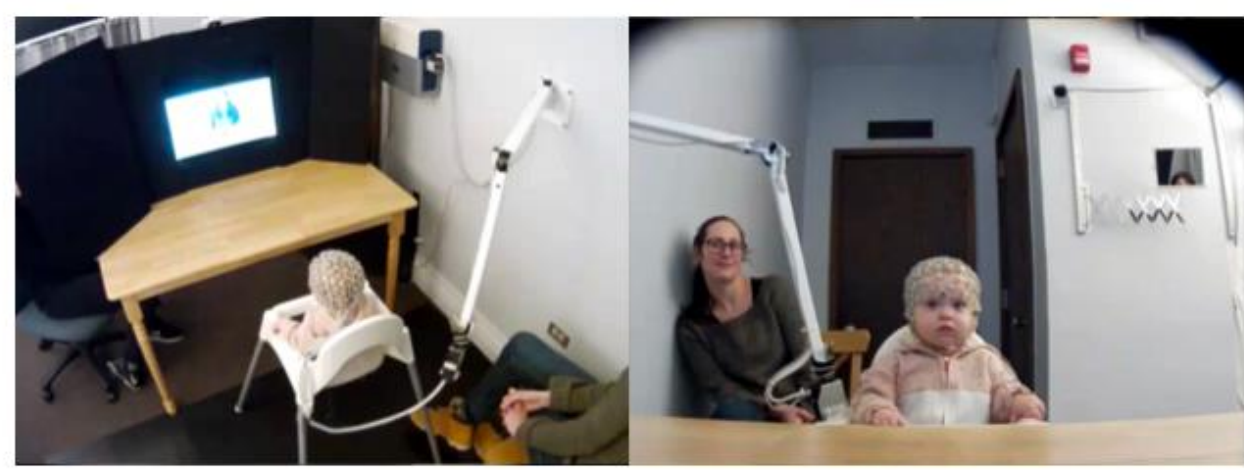

Figure 1 [Color should be used for print]. A. Schematic time-line of an observation trial. (a) grasp condition; (b) cane-use condition. B. Experimental room set-up of the observation phase. Left: Example of an infant participant and parent in the set-up during the observation phase facing the computer screen. Right: View of infant and parent during the observation phase.

\section{Action observation}

During the action observation phase, two types of actions were presented: a familiar action (i.e. grasping a toy to bring it closer) and a novel tool-use action (i.e. using a cane to bring a toy closer; see Figure 1). Both action observation conditions were presented with 4000-4500ms 
long videos. To make the transition of the videos look more natural, we put a dissolve filter in the video editing software (Adobe Premiere Pro) that introduced a gradation of brightness for $500 \mathrm{~ms}$ (dark to bright) at the start and end of each video for both conditions. Each video began with the experimenter sitting still and facing the center of the screen and saying "Hey, Baby!" or "Hi there!", presented in a pseudo-random order with no more than 3 repetitions in order. The toy for the grasp condition and the toy and cane for the cane-use condition were present in the scene from the start of the videos. In the grasp condition, the experimenter looked down at the toy, grasped it and brought the toy closer. In the cane-use condition, the experimenter looked down at the toy and then pulled the cane to bring the toy closer. Before each action observation video, a still image of the experimenter sitting in front of an empty table was presented for $1000 \mathrm{~ms}$. Trials were presented in a semi-randomized order of grasping and caneuse action videos, such that infants were presented with a maximum of 20 trials per set, up to 40 trials in total. The order of the conditions was pseudorandomized so that infants did not observe more than three trials of the same type of action in a row. Ten unique toys were used for the two types of action conditions such that all toys appeared twice for each trial type. Infants completed a mean of 33.4 trials (SD=7.1, Range: $16-40),(9 \mathrm{~m}: 32.7$ trials, $S D=6.7 ; 12 \mathrm{~m}$ : 34.1 trials, $S D=7.5)$. After 40 trials or once the infants lost interest, the observation phase ended and infants participated the action execution phase.

\section{Action execution}

We collected infants' execution of grasping and cane use action to A) confirm whether the neural response during action observation indeed reflects the neural response during the execution of action and B) to examine whether individual variance in motor competence affects infants' processing of action. During the action execution phase, infants sat in front of a table (see Figure 2). Across the table, E1 positioned herself behind curtains and E2 sat next to the caregiver, outside of the infants' visual scene. The execution phase began with two warm-up trials, where $\mathrm{E} 1$ (the same person infants observed in the videos during the action observation phase) opened the curtains, saying "Hi, baby. Are we ready?" and closed the curtains. These warm-up trials were to ensure that the infants were attending and ready to proceed with the 
session. Every infant received 5 grasp trials and 5 cane-use trials in a fixed order, first grasp followed by cane-use trials. We utilized a fixed order of grasp and cane-use execution trials to be sure to get at least infants' execution of grasps. The execution grasp condition began with E1 opening the curtains, not moving for $1000 \mathrm{~ms}$ (baseline), and then closing the curtains. Then, E1 opened the curtain again and said "Hey baby, look!" showing the toy to the infant. E1 placed the toy in front of the infant, making sure that the toy was placed within the infants' reach. The execution grasp condition ended when the infant grasped the toy. If the infant did not attempt to grasp the toy within 10 seconds, E1 pointed or tapped the toy so that the infant attended to the toy. Once infants reached for and grasped the toy, or after 3 repetitions of the prompt, E1 retracted the toy and closed the doors. Every trial ended with E1 providing positive encouragement (i.e. "Good job" or "All right"). Infants completed a mean of 4.74 trials (SD= 0.94, Range: $1-5)$, (9m: 4.8 trials, $S D=0.91 ; 12 \mathrm{~m}: 4.68$ trials, $S D=0.98)$.

The execution cane-use condition also began with the same baseline prompt as the grasp execution condition. Then, E1 opened the curtain and said "Look" showing the toy to the infant, and placed the toy at the end of the table, beyond infants' reach. E2 then placed the cane (length of cane: $48 \mathrm{~cm}$, width of crook: $13.5 \mathrm{~cm}$ ) around the toy. If the infant did not attempt to grasp the toy within 10 seconds, E1 pointed or tapped the toy so that the infant attended to the toy. E1 did not point to the cane nor provided any assistance. The cane-use trial ended after infants either successfully retrieved the toy by pulling the cane or 60 seconds elapsed. Every trial ended with E1 providing positive encouragement (i.e. “Good job" or "All right"). Infants completed a mean of 4.07 trials $(S D=1.2$, Range:1-5), (9m: 4.14 trials, $S D=1.2 ; 12 \mathrm{~m}: 4$ trials, $\mathrm{SD}=1.3)$.

At the end of the testing session, caregivers were debriefed about the goal of the study and families received a small gift (toy or t-shirt) and 20 US dollars as compensation for participating in the study. 
Neural Correlates of Familiar and Unfamiliar Action in Infancy

\section{Execution phase}

\section{A. Schematic time-line of Execution trial}

\section{(a)Grasp Condition}

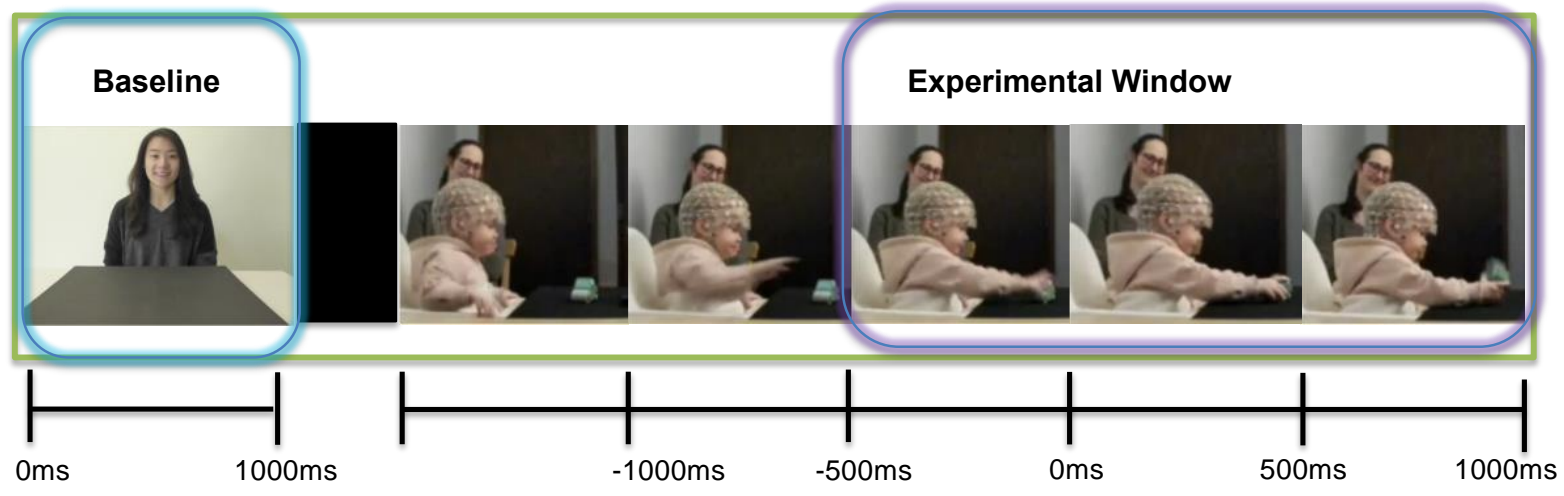

(b)Cane-use Condition

*0- time locked to first touch of toy / cane

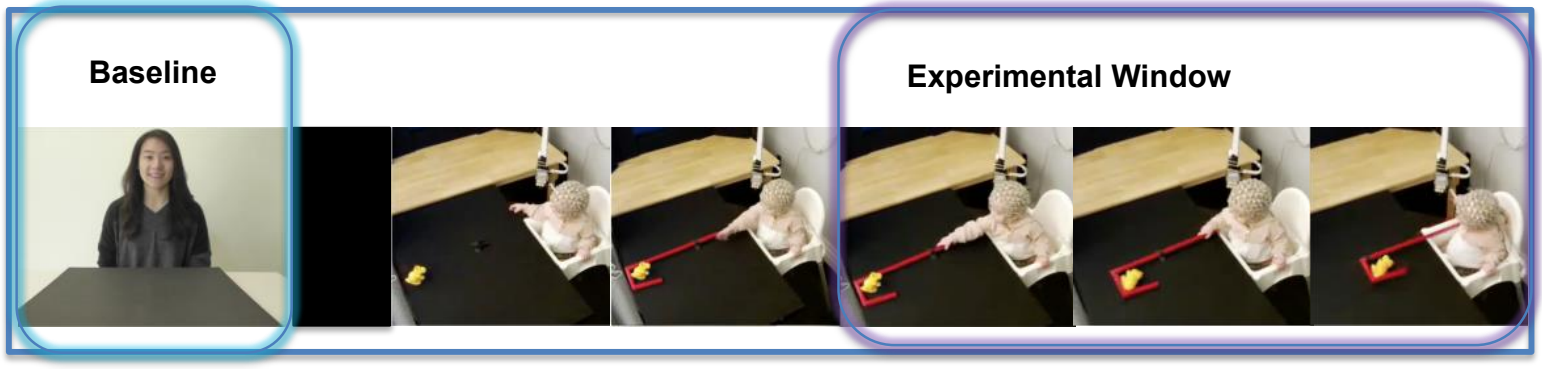

B. Experimental set up during Execution phase

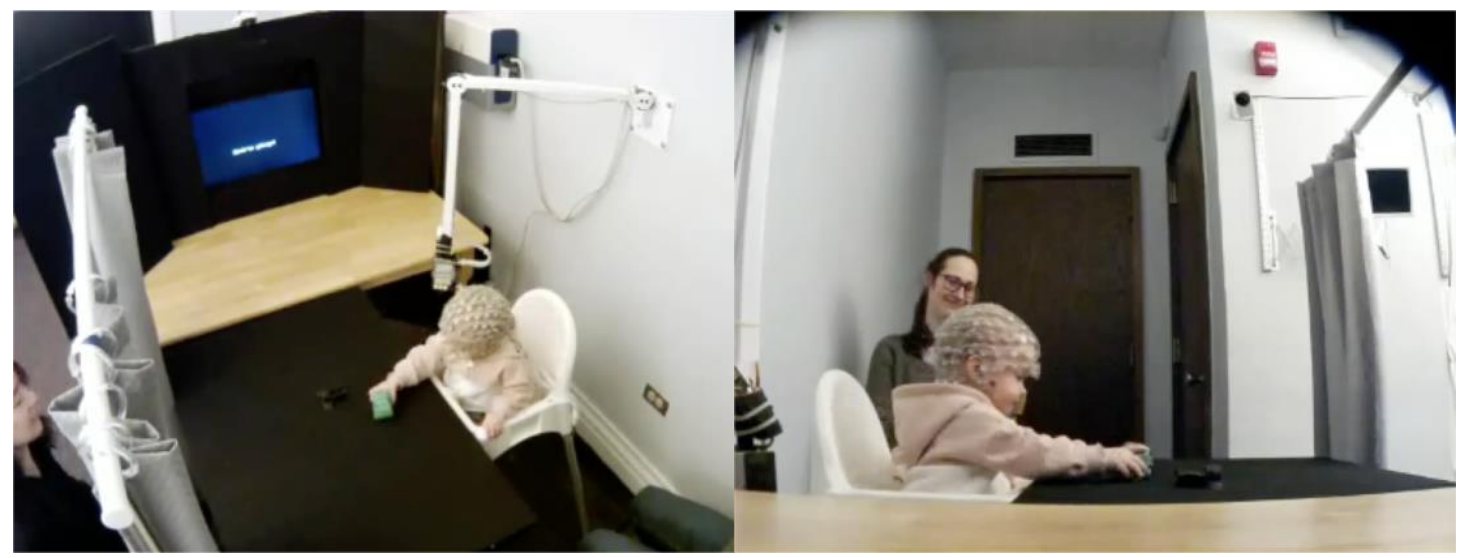

Figure 2 [Color should be used for print]. A. Schematic time-line of an execution trial. (a) grasp condition; (b) cane-use condition. B. Experimental room set-up of the execution phase. Left: View of infant and E1 during an execution grasp trial (E1 left to the curtain). Right: View of infant and parent during execution grasp trial. 
Neural Correlates of Familiar and Unfamiliar Action in Infancy

\section{Analysis}

\section{Video-coding analysis}

An open-source video coding software Datavyu (Datavyu Team, 2014) was used for coding videos offline. It was important that we utilize a baseline that closely matches the visual scenes of the action observation experimental window of each condition in order to reduce the influence of visual differences. The baseline for action observation phase was coded as the first frame after change of brightness (from dark to bright), and when E1 was visible with objects (toy and cane) present, but not moving for 500ms (Figure 1). The baseline period included the experimenter sitting still and greeting the child without further movement. The baseline did not include any arm or hand movements and this was the same across conditions. The experimental time window in the action observation phase was coded from the first frame of E1's movement onset (started to reach in grasp condition or started pulling the cane in caneuse condition). E1's onset of movement was time-locked to 0ms, and data of 1000ms before and $1000 \mathrm{~ms}$ after this mark were taken as the experimental time window for the analyses. For execution trials, since it was impossible to match the visual scene of the baseline and experimental windows for execution trials, the baseline was coded as the first frame that the curtains were fully open, and when E1 was visible to the infant for $1000 \mathrm{~ms}$. The experimental time window for the grasp and cane-use condition was coded based on the first frame that the infant touched the toy and the contact led to grasping the toy / that led to pulling the cane. Infants' touch was time-locked to $0 \mathrm{~ms}$, and data of $500 \mathrm{~ms}$ before and $1000 \mathrm{~ms}$ after this mark was taken as the time window for EEG execution analyses (Figure 2).

In order to capture infants' processing of others' actions in the action observation phase and not the coincidental execution of their own actions, we coded moments of infants' own grasping actions, which included grasping (e.g., arm of the chair, or mother's hand) or grasping movements without an object (e.g., making a grasping movement in the air) in the action observation phase. All action observation and baseline EEG segments that overlapped with parental interference (e.g., talking to the infant, bouncing the infant), crying, infant not-looking 
Neural Correlates of Familiar and Unfamiliar Action in Infancy

at the screen, or infants' own grasping were excluded from further analysis (see supplemental material for analysis of gross and fine-motor movements).

\section{Behavioral Analysis}

\section{Grasping latency}

Based on Cannon et al. (2016) and Yoo et al. (2016), we examined infants' grasping competence during execution trials. We assessed the latency with which infants grasped the toys as a marker of their grasping competence. The amount of time was calculated from the touch that resulted in a grasp until the completion of the grasp. Grasp completion was coded as the beginning of the toy pick up or infants' fingers wrapped around the toy if there was no pick up. Only trials with touches that led to a toy grasp were included to calculate the grasp latency measure. An independent coder coded $73 \%$ of the data. A high degree of reliability was found (ICC- 0.78 ) with $95 \%$ confidence interval from 0.57 to 0.9 for grasping latency. Infants had to provide at least two codable trials to be included in the analysis.

\section{Cane pulling latency}

In order to use a measure comparable to the grasp latency measure, we defined infants' caneuse competence during execution trials as latency to pull the cane. The average amount of time was calculated from the pull that resulted with the toy within infants' reach. A successful pull in the cane-use condition was defined as 1 ) looked at the toy being placed at the end of the cane 2) initiated one clear pull without removal of hand from cane (until the cane reached the endpoint of the rack) and 3) maintained eye-gaze throughout the pull. An independent coder coded $70 \%$ of the data. A high degree of reliability was found (ICC- 0.95 ) with $95 \%$ confidence interval from 0.86 to 0.98 for cane-pulling latency. Infants had to provide at least two codable trials to be included in the analysis.

\section{EEG data analysis}


The EEG data preprocessing was performed by the Maryland analysis of developmental EEG (MADE) pipeline (Debnath et al., 2020). Recordings were converted and exported to a Matlabcompatible format (The Mathworks, Natick, MA) using Net Station software. EEG data (pre)processing was conducted using EEGLAB (v14.0.0) toolbox (Delorme \& Makeig, 2004). First, video-coded markers were imported onto the EEG dataset and data were downsampled to $500 \mathrm{~Hz}$. The subsequent processing steps are identical to those applied in Debnath et al., (2019) and Meyer et al., (2022). The outer ring of electrodes on the 128-channel net have often poor connections in pediatric data (see Debnath et al., 2020 for more detail) and were therefore removed a priori (E17, E38, E43, E44, E48, E49, E113, E114, E119, E120, E121, E125, E126, E127, E128, E56, E63, E68, E73, E81, E88, E94, E99, E107) with 104 channels remaining for analysis. The continuous data were filtered at $0.3 \mathrm{~Hz}$ highpass and $50 \mathrm{~Hz}$ lowpass using FIR filter provided within the FIRfilt plugin of EEGLAB (Widmann et al., 2015). Then, using FASTER (Nolan et al., 2010) plugin of EEGLAB bad channels were detected and removed. In order to filter out non-neural artifacts including blinks, saccades, or EMG, we applied a hybrid approach of using a copied dataset, applying a $1 \mathrm{~Hz}$ high-pass filter and segmenting data into 1-second epochs, removing excessive EMG, and then running ICA on the copied dataset as suggested by Debnath et al. (2020). Then ICA weights were applied to the original dataset. A semi-automatic process using ADJUST EEGLAB plugin (Mognon et al., 2011) identified ICA components and the components were also manually reviewed. Next, time-windows of interest (baseline and observation trial segments) that were contaminated by the infant grasping, not looking, crying or the parent interfering were excluded from further analysis based on video coding. The remaining data were segmented into baseline segments and experimental segments. A voltage threshold rejection $( \pm 150 \mu \mathrm{V})$ was applied on six frontal channels (E1, E8, E14, E21, E25, E34) to identify and remove additional eye artifacts. For all other channels, we interpolated data for artifacted channels in each epoch. Epochs with more than $10 \%$ of interpolated channels were rejected. After preprocessing, 3 participants were excluded in the observation condition from further time-frequency and connectivity analysis because they had an insufficient number of artifact free trials ( $<3$ in each condition). For the execution condition, 12 participants for grasp and 19 participants in the cane-use condition were excluded from further time-frequency and 
Neural Correlates of Familiar and Unfamiliar Action in Infancy

connectivity analysis because they had an insufficient number of artifact free trials ( $<3$ in each condition, following minimum trial requirement of infant mu rhythm studies, Marshall et al., 2011; Debnath et al., 2019). (See supplemental material for a detailed breakdown of inclusion of participants for EEG analysis). For the observation session, there were a mean of 8.3 artifact free grasp trials (range 3-17) and a mean of 7.5 artifact free cane-use trials (range 3-19) in the final 9-month-old dataset, and a mean of 9 artifact free grasp trials (range 4-19) and a mean of 10.4 artifact free cane-use trials (range 4-19) in the 12-month-old dataset. For the execution session, there were a mean of 4.3 artifact free grasp trials (range 3-5) and a mean of 3.7 artifact free cane-use trials (range 3-5) in the 9-month-old dataset, and a mean of 3.1 artifact free trials (range 3-5) in the grasp condition and a mean of 3.3 artifact free cane-use trials (range 3-5) in the 12-month-old dataset. Preprocessed epoch data were then converted into current source density (CSD) using the CSD toolbox (Kayser \& Tenke, 2006), and event-related spectral perturbation (ERSP) was calculated on CSD transformed data to estimate the baselinecorrected spectral power (in $\mathrm{dB}$ ) between 3- to $30 \mathrm{~Hz}$ for all channels and trials. (The derived EEG data in BIDS format and Matlab analysis scripts are available on the Open Science Framework: https://osf.io/f9da5/).

Time-frequency analysis

Time-frequency decomposition was computed using the EEGLAB newtimef function. To assess infants' neural motor activity during action observation and execution consistent with previous research (Debnath et al., 2019; Marshall et al, 2011; Meyer et al., 2022), our analyses focused on mu power, by measuring baseline-corrected spectral power in the $6-9 \mathrm{~Hz}$ alpha frequency band in channels overlaying sensorimotor (Central) regions (C3 = E29, E30, E35, E36, E37, E41, E42; C4 = E87, E93, E103, E104, E105, E110, E111; Figure 3). We did not include the midline channel $(\mathrm{Cz})$ because the activity in $\mathrm{Cz}$ is found to be associated with and modulated by leg movements in infants (van Elk et al., 2008; de Klerk et al., 2016) while C3 and C4 are found to be associated with hand and arm movements in infancy (Saby et al., 2013). Mu power during action observation was calculated for the following time windows: anticipatory $(-1000 \mathrm{~ms}$ to Oms) time window during which E1 is still and no movement is happening, and movement (0ms 
Neural Correlates of Familiar and Unfamiliar Action in Infancy

to $1000 \mathrm{~ms}$ ) during which E1 starts to produce the action (grasp condition: 0 to $500 \mathrm{~ms}$ entails E1's reach to the toy, and 500 to 1000 ms entails the E1's grasp completion of bringing the toy closer to her; cane-use condition: 0 to 1000ms entails E1's cane pulling action). Mu power during action observation was calculated relative to the experimenter present with the object but without movement. Mu power during execution was calculated for infants' action onset ($500 \mathrm{~ms}$ to $0 \mathrm{~ms}$ and $0 \mathrm{~ms}$ to $1000 \mathrm{~ms}$ ) relative to the experimenter present without presence of objects. In addition, power over frontal, parietal, and occipital regions was analyzed as well (F3= E19, E20, E23, E24, E27, E28; F4=E3, E4, E117, E118, E123, E124; P3=E47, E51, E52, E53, E59, E60; P4=E85, E86, E91, E92, E97, E98; O1=E66, E69, E70, E71, E74; O2=E76, E82, E83, E84, E89; see Figure 3).

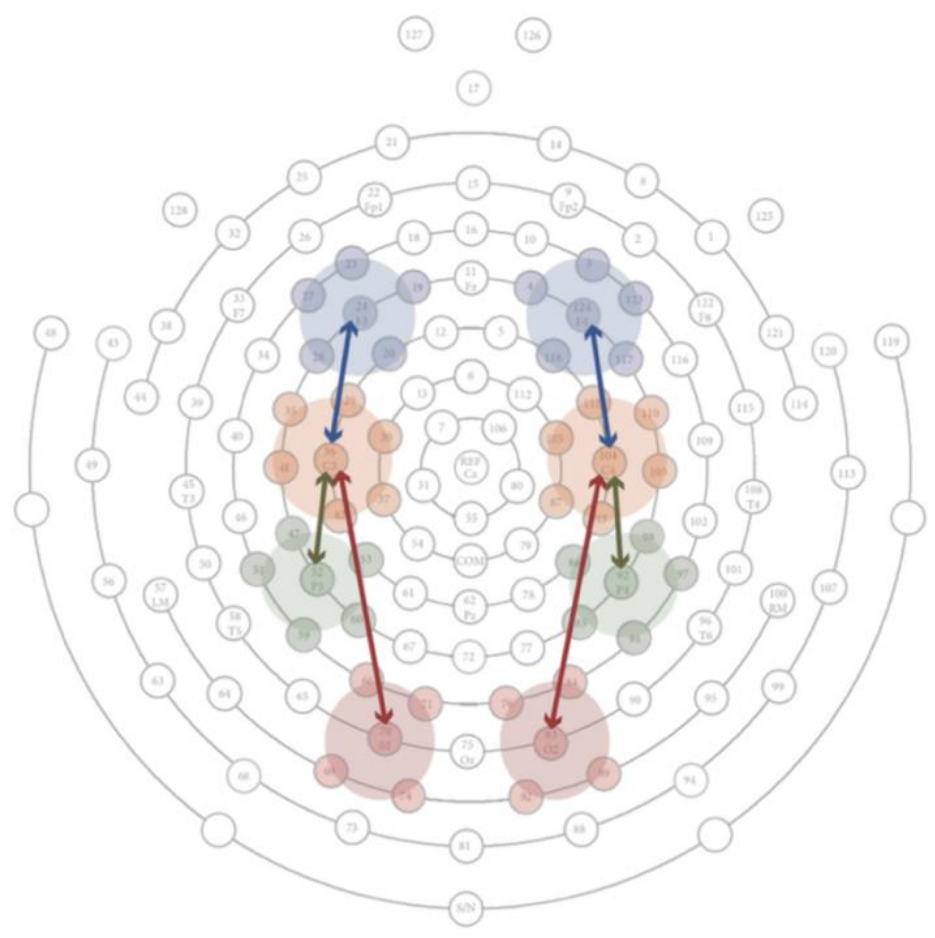

\section{A. Channel Clusters}

Frontal

Central

Parietal

Occipital

B. ICPC Channel Cluster Pair

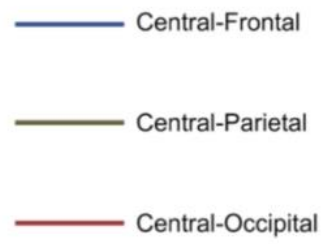

Figure 3 [Color should be used for print]. A. Electrode channel clusters, from top to bottom, for Frontal (Blue circle), Central (orange circle), Parietal (green circle), and Occipital (red circle) scalp locations. B. Arrows indicating ICPC channel cluster pair between regions. Blue arrow: Central-Frontal, Green arrow: Central-Parietal, Red arrow: Central-Occipital.

Statistical comparison across time and frequencies 
Neural Correlates of Familiar and Unfamiliar Action in Infancy

We performed point-wise analyses of spectral power modulation in all electrodes for each time window against a null hypothesis of no change in baseline-corrected power (represented by zero) during the event of interest. We computed one sample non-parametric permutation tests with FDR correction for multiple comparisons against zero for each time point using the "std_stat" function of the EEGLAB toolbox for visualization.

Inter-channel phase coherence

We measured coherence between brain regions by calculating inter-channel phase coherence (ICPC), which estimates the consistency of phase angle difference between two clusters of channels over time and frequency (Cohen, 2014). Phase angles were first calculated from two clusters of electrodes (see below) and then subtracted. ICPC value (range: 0 to 1 ) closer to 1 is interpreted as more synchrony between two channel clusters, supporting the idea that when brain regions are functionally coupled, the timing of their oscillatory processes becomes synchronized (Cohen, 2014). As in Debnath and colleagues (2019), we calculated ICPC between channel clusters over central areas and the three other brain areas in both the left (C3- F3, C3P3, C3- 01) and right (C4- F4, C4- P4, C4- O2) hemispheres (See Figure 3). We also calculated ICPC between occipital and the three other brain regions in both hemispheres (left: O1- F3, O1- C3, O1- P3; right: O2- F4, O2- C4, O2- P4) (See supplemental material). We computed ICPC in both observation of grasping and cane-use conditions at 6-9 Hz frequency during movement time window (0ms to $500 \mathrm{~ms}$ time-locked to movement onset). 
Neural Correlates of Familiar and Unfamiliar Action in Infancy

\section{Results}

\section{Mu suppression during action execution}

We first assessed neural activity during action execution with the aim to verify that 6-9Hz range covers neural processing of execution of grasping and cane-pulling action in this sample of infants. There was significant $6-9 \mathrm{~Hz}$ mu power suppression in the electrode clusters overlying the motor cortex both during execution of grasping and cane-pulling action. Thus, suppression of power in the $6-9 \mathrm{~Hz}$ frequency range captures action processing in this sample and can therefore serve as an index of neural motor activation in the observation phase. (For more detailed information on action execution, see supplemental materials).

\section{Mu and occipital alpha during observation of action}

We analyzed 36 (9m-17, 12m-19) participants' neural activity during action observation. Figure 5 displays results of comparing alpha power in $6-9 \mathrm{~Hz}$ frequency range relative to baseline over frontal, central, parietal, and occipital electrode clusters in each condition during the observation phase collapsed over age. All activity shown is significantly different from zero at the $p<.05$ level with FDR correction for multiple comparison.

The point-wise analysis revealed that there was significant mu suppression in the electrode clusters overlying the motor cortex $(\mathrm{C} 3, \mathrm{C} 4)$ both prior to the start of the E1's movement ($1000 \mathrm{~ms}$ to $0 \mathrm{~ms}$ ) and continuing through the movement period for both grasp and cane-use conditions for both age groups (0ms to 500ms) (Figure 4, Central). Significant suppression over the occipital region was evident around the onset of the E1's movement in the grasp condition (Oms to $1000 \mathrm{~ms}$ ), but in the cane-use condition, occipital alpha was significantly suppressed from around $500 \mathrm{~ms}$ post-movement observation (Figure 4, Occipital). (Verification that mu power and occipital alpha, measured using the baseline in our current study, are reflecting independent neural activity and likely come from different sources is reported in supplemental material). 
(a)Grasp Condition

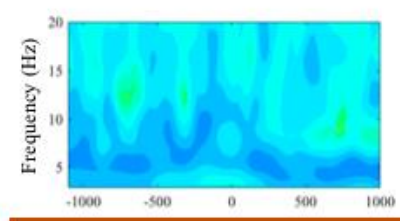

(b)Cane-use Condition
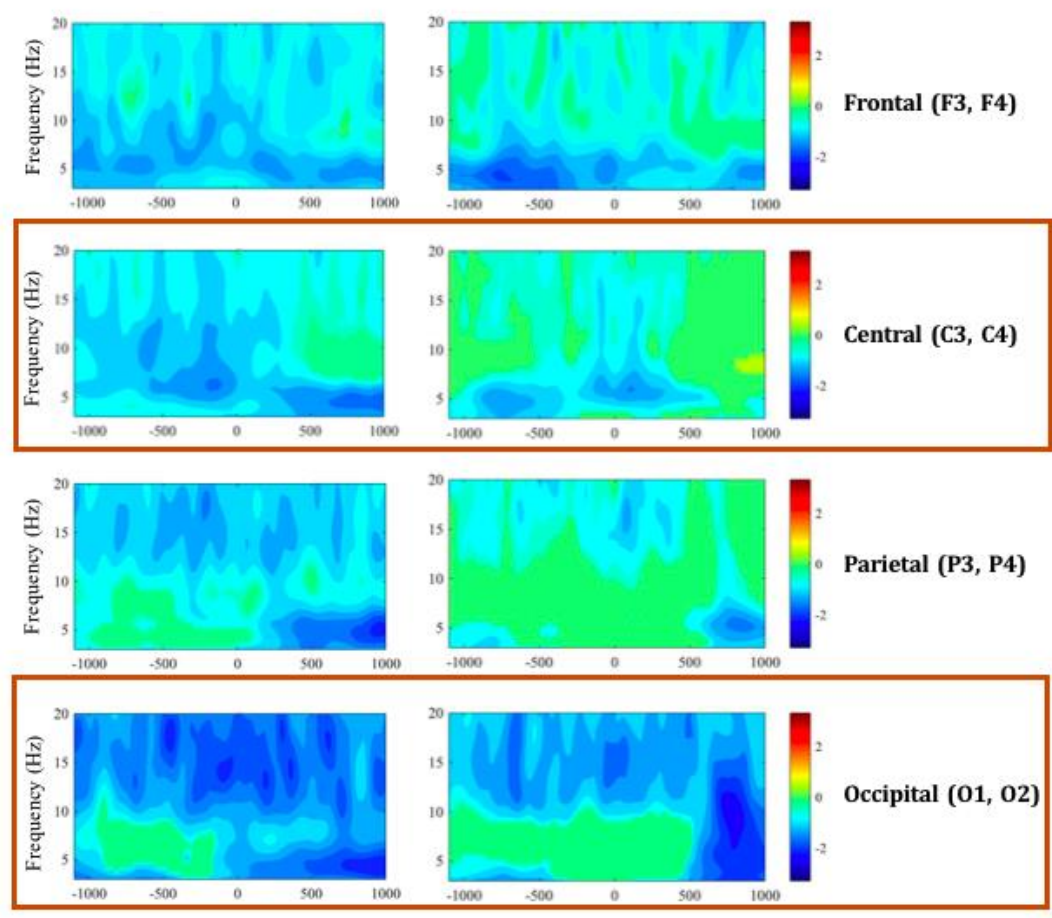

Figure 4 [Color should be used for print]. Statistically significant time-frequency plot of spectral power relative to baseline during observation phase for (a) grasp condition, (b) cane-use condition, across age. The color towards blue indicate significant suppression of power relative to baseline. Green in images depicts non-significant $(p>.05)$ activity. Power (decibels, $d B)$ is shown by the color bar.

\section{Mu/occipital alpha suppression and motoric familiarity}

To address our main question of whether motor familiarity influences mu power and occipital alpha, we performed a 4-way mixed ANOVA with condition (grasp, cane-use) and time (anticipatory: $-1000 \mathrm{~ms}$ to $0 \mathrm{~ms}$; movement: $0 \mathrm{~ms}$ to $1000 \mathrm{~ms}$ ) and hemisphere (left, right) as within-subjects variables, and age $(9 \mathrm{~m}, 12 \mathrm{~m})$ as between-subject variable for each region. We included two time windows due to visual differences across time in the time-frequency plot (Figure 4, central and occipital). For the model with central region, we found a significant main effect of condition $(F(1,34)=5.0, p=.03)$ and a significant main effect of time $(F(1,34)=1.69, \mathrm{p}$ $<.001)$. Follow-up analyses were performed to determine the effects of condition and time. 
Neural Correlates of Familiar and Unfamiliar Action in Infancy

Pairwise t-test between time windows (anticipatory and movement) revealed a significant difference on mu power $(t(35)=-3.9, p<.001)$, with more mu power suppression observed during the anticipatory period $(M=-.91 \mathrm{~dB}, \mathrm{SE}=.17)$ than during movement $(\mathrm{M}=-.51 \mathrm{~dB}, \mathrm{SE}$ $=.18)$. Pairwise t-test between condition revealed that there was more overall mu power during observation of grasp action $(\mathrm{M}=-.90 \mathrm{~dB}, \mathrm{SE}=.16)$ than for cane-use action $(\mathrm{M}=-.52 \mathrm{~dB}$, $\mathrm{SE}=.2),(t(35)=-2.3, p=.03$, Cohen's $d=0.29$; Figure 5).

For the model with occipital region, we found a significant main effect of time $(F(1,34)=25.2, p$ $<.001)$, a significant interaction of time and age $(F(1,34)=9.3, p=.05)$, and a significant threeway interaction of time, age, and hemisphere $(F(1,34)=4.42, p<.001)$. Follow-up pairwise ttest between time windows revealed a significant difference on occipital alpha $(t(35)=4.67, p$ $<.001)$, with more occipital alpha suppression observed during the movement $(\mathrm{M}=-1.02 \mathrm{~dB}, \mathrm{SE}$ $=.17)$ than anticipatory period $(M=-.37 \mathrm{~dB}, \mathrm{SE}=.20)$. The interaction effect of age and time resulted from stronger occipital alpha during movement in 12-month-olds $(\mathrm{M}=-1.35 \mathrm{~dB}, \mathrm{SE}$ $=.23)$ than during anticipatory period in 12-month-olds $(M=-.35 \mathrm{~dB}, \mathrm{SE}=.19),(t(35)=5.88, \mathrm{p}$ $<$.001). No other pair was significant after Bonferroni correction. A follow-up Bonferroni posthoc t-test on the 3-way interaction was conducted and showed that there was significant difference between anticipatory left $(M=-.39 \mathrm{~dB}, \mathrm{SE}=.34)$ and movement left $(\mathrm{M}=-1.34 \mathrm{~dB}, \mathrm{SE}$ $=.27)$ in 12 -month-olds $(t(18)=5.36, p<.001)$ and significant difference between anticipatory right $(M=-.30 \mathrm{~dB}, \mathrm{SE}=.29)$ and movement left in 12-month-olds $(\mathrm{t}(18)=5.69, \mathrm{p}<.001)$. Importantly, in contrast to mu power, occipital alpha power was not modulated by condition.

Together, we found that mu suppression was evident for observation of both familiar and unfamiliar action. This is in line with previous work on infant mu rhythm during observation of grasp action (Debnath et al., 2019; Meyer et al., 2022; Southgate et al., 2009; Hwang et al., 2021), and for novel action (Southgate \& Begus, 2013; Virji-Babul et al., 2012). Importantly, greater mu suppression was exhibited during observation of grasp than cane-use action. This provides evidence that infants' mu suppression is modulated by motoric familiarity. 
Neural Correlates of Familiar and Unfamiliar Action in Infancy

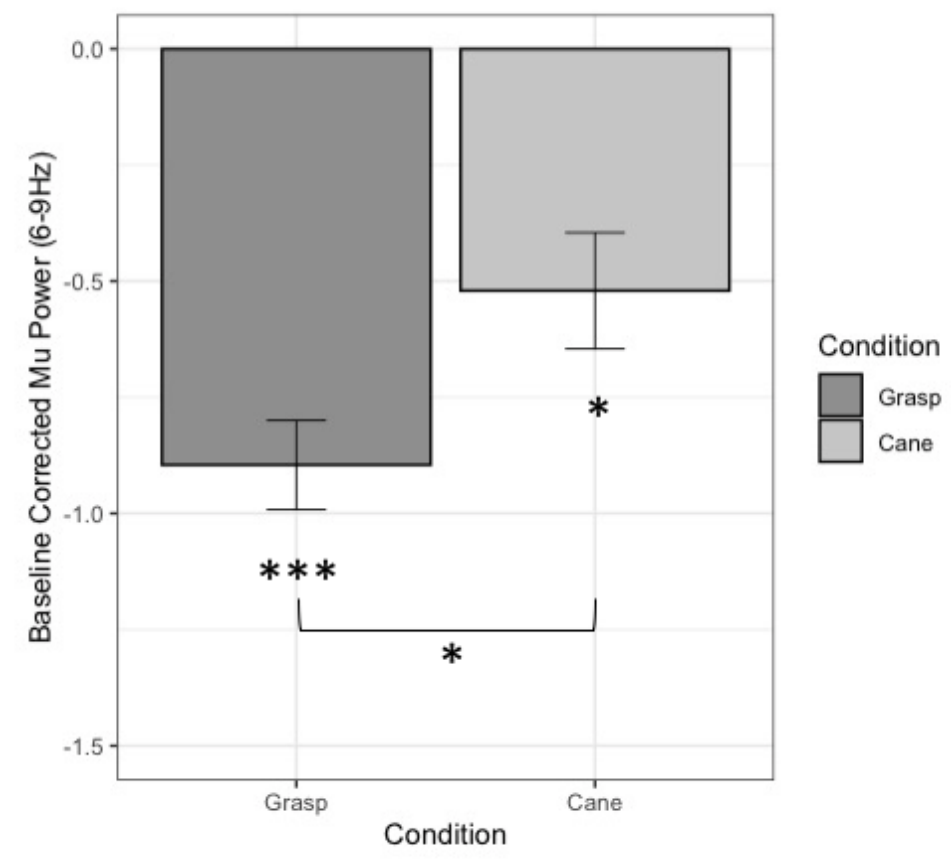

Figure 5. Baseline-corrected power of the alpha frequency band $(6-9 \mathrm{~Hz})$ over Central $(\mathrm{C} 3, \mathrm{C} 4)$ sites as a function of condition (Grasp / Cane-use). Error bar indicates \pm 1 SE. $* p<.05, * * * p$ $<.001$.

\section{Mu/alpha suppression and behavioral competence}

13 9-month-olds and $1712 \mathrm{~m}$-month-olds had sufficient grasp behavioral execution trials, and 10 9-month-olds and $1112 \mathrm{~m}$-month-olds had sufficient cane pulling behavioral execution trials. The mean grasp latency was $846 \mathrm{~ms}(S D=400)$ for 9 -month-olds and $775 \mathrm{~ms}(S D=280)$ for 12 month-olds. The mean cane pulling latency was $2158 \mathrm{~ms}(S D=760)$ for 9 -month-olds and $1749 \mathrm{~ms}(\mathrm{SD}=978)$ for 12 -month-olds. We found no differences between the two age groups for grasp ( $p>0.5)$ and cane pulling ( $p>0.3$, Figure 6$)$ latencies.

To investigate whether mu suppression is associated with behavioral efficiency with an action, we submitted the competence measure (latency to grasp, latency to pull) as our dependent variable and mu power during observation of action (0ms to $1000 \mathrm{~ms}$ ) and age $(9 \mathrm{~m} ; 12 \mathrm{~m})$ as independent variables for each condition in separate regression models. In the grasp condition, 
Neural Correlates of Familiar and Unfamiliar Action in Infancy

the model with age and mu power revealed a marginally significant model, $R^{2}=.26, F(3,24)=$ $2.74, p<.06$, and a significant interaction of mu power and age group, $(b=-322.6, S E=117.5, t$ $=-2.75, p=.01)$, and a significant main effect of mu power $(b=248, S E=96.3, t=2.58, p=.02)$ on latency to grasp (Figure 7). Follow-up analyses were performed to determine where the interaction effect originated. We found a positive correlation between mu power and grasp latency in 9-month-old group $(r=-.59, p<.04)$, with faster grasping latency associated with more suppression in mu power. We found no evidence of such relation in the 12-month-old group $(r=-.31, p>.2)$, possibly due to less variability in the 12-month-old grasping latency to detect meaningful relation. In the cane-use condition, we did not find evidence for a relation of mu power and age on infants' cane pulling latency measure $(p>.1)$. We also conducted the same analysis with occipital alpha and found no evidence of a relation between occipital alpha and behavioral competence (grasp latency: $p>0.8$; cane pulling latency: $p>0.5$; correlation between occipital alpha and behavioral competence: $B_{01}=4.1, B_{01}=3.0$, respectively). These findings suggest that motor experience with a familiar action is uniquely associated with infants' mu suppression during action observation, as has been found in prior studies (Cannon et al., 2016; Gerson, Bekkering et al., 2015; Paulus et al., 2012; van Elk et al., 2008). No such correlation was evident for the novel tool-use action.

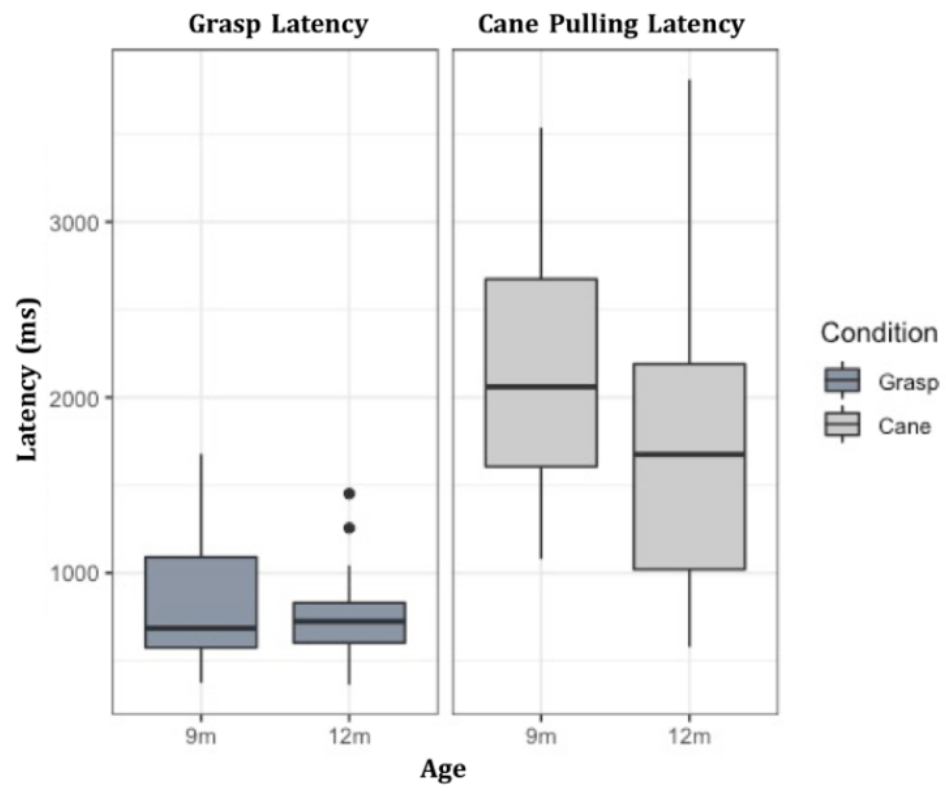


Figure 6 [Color should be used for print]. Bar-plot of infant' behavioral competence. Left: Infants' grasping latency, Right: Infants' canepulling latency, in milliseconds.
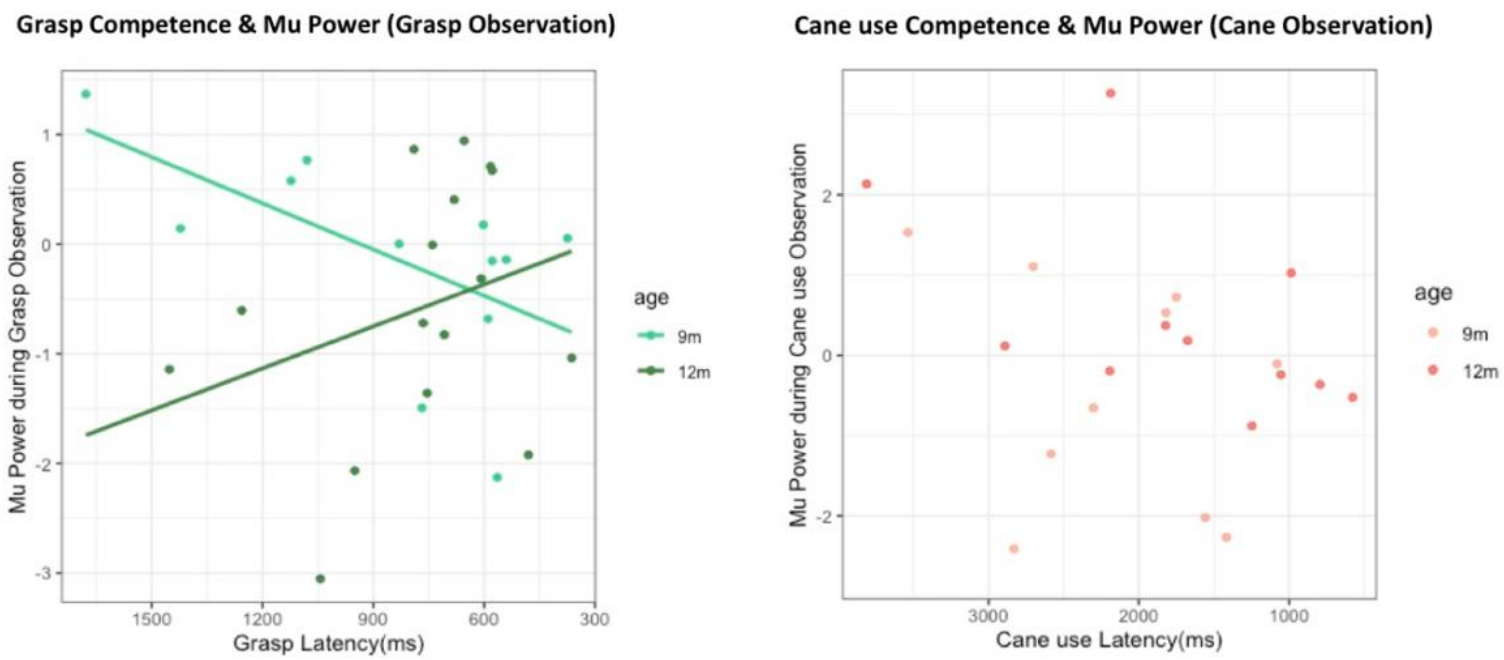

Figure 7 [Color should be used for print]. Scatter plot of Mu Power (6-9Hz) overlying central area (a) during observation of grasping action and infants' grasp latency (in milliseconds), and (b) during observation of cane-use action and infants' cane pulling latency. X-axis scale is reversed, indicating faster latency towards right side of the axis. (Note: (a) only the regression line for $9 \mathrm{~m}$ is significant; the line for $12 \mathrm{~m}$ is not significant)

\section{Inter-channel phase coherence}

To explore functional connectivity of motor and visual processes, we compared ICPC at 6-9 Hz frequency for electrode pairs between central-occipital to that of central-frontal and centralparietal. We analyzed 36 (9m-17, 12m-19) infants' ICPC. Our goal in exploring ICPC was twofold. First, we tested whether we replicate the findings by Debnath et al. (2019) for the grasp condition. Second, we wanted to explore the pattern of ICPC for a novel cane-use action. We analyzed the ICPC in a 3-way mixed ANOVA with age $(9 \mathrm{~m}, 12 \mathrm{~m})$ as a between-subject factor and hemisphere (right, left) and electrode-pair (central-frontal, central-parietal, central-occipital) as within-subject factors independently for each condition (grasp, cane-use). 
For ICPC in the grasp condition, the analysis revealed a significant main effect of electrode-pair $(F(2,68)=5.12, \mathrm{p}<.05)$, and a significant interaction effect of hemisphere and age $(F(1,68)=$ $4.3, p<.05$; partial eta squared $=0.14$ ). Follow up pair-wise comparisons after Bonferroni correction revealed that ICPC between central-occipital electrodes pair $(\mathrm{M}=.39, \mathrm{SE}=.08)$ was significantly higher than central-frontal $(\mathrm{M}=.36, \mathrm{SE}=.08 ; \mathrm{t}(35)=2.56, \mathrm{p}=.01)$ and centralparietal $(M=.36, S E=.07 ; t(35)=2.62, p=.02)$ (Figure $8(a))$. For the interaction effect of hemisphere and age, no pairs were significant after Bonferroni correction; however, the interaction effect seemed to be originated from differences in 12-month-olds. Descriptively, there was higher connectivity in the left $(M=.37, S E=.01)$ than right hemisphere $(M=.35, S E$ $=.01$ ) in the 12-month-olds across pairs. In the cane-use condition, we did not find evidence for a difference between electrode-pair connectivity (Figure 8(b)). Thus, we replicate the findings in Debnath et al., (2019) for the grasp condition and confirm that motor and visual areas are functionally connected during observation of familiar, grasping action. We find no evidence for a difference of connectivity among regions during observation of a novel, tool-use action. (See supplemental material for ICPC regarding connectivity between occipital-frontal, occipitalparietal, occipital-central).

\section{Central ICPC (Observation during Movement)}

(a)Grasp Condition

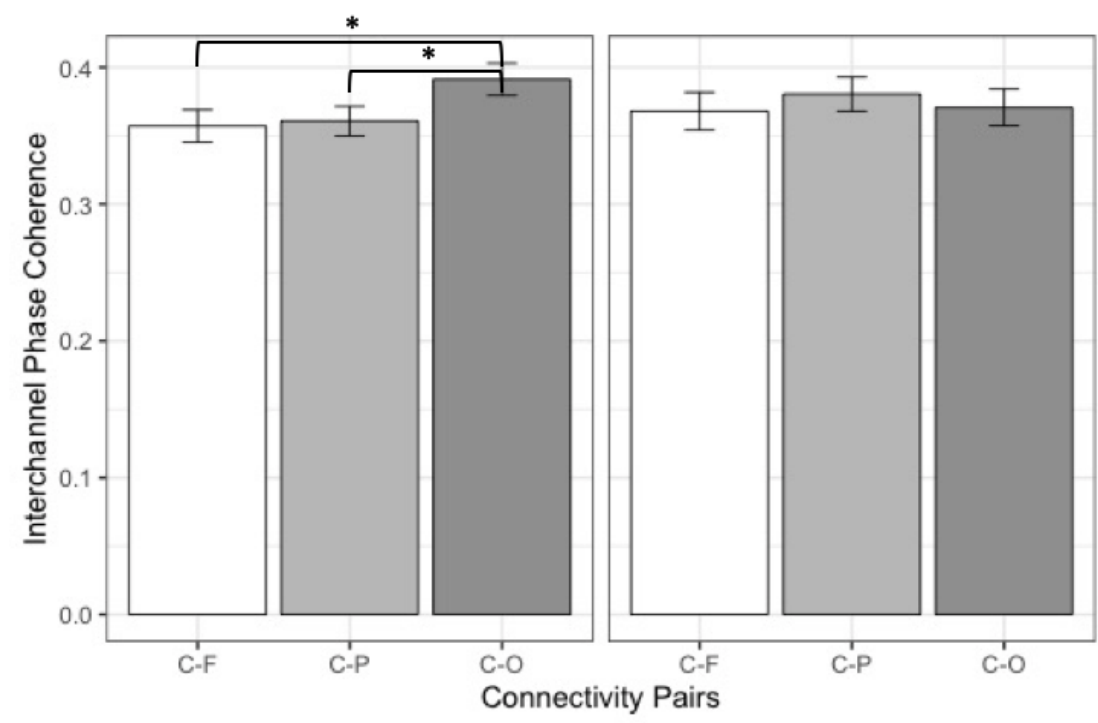

Electrode Pair

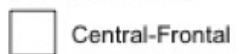

Central-Parietal

Central-Occipital 
Neural Correlates of Familiar and Unfamiliar Action in Infancy

Figure 8. Average of central-based Inter-channel phase coherence (ICPC) between channels pairs over frontal, central, parietal and occipital areas in 6-9 Hz frequency band. The time window includes Oms to 500ms during observation of (a) grasp and (b) cane-use action. Error bar indicates \pm 1 SE. ${ }^{*} p<.05$. 
Neural Correlates of Familiar and Unfamiliar Action in Infancy

\section{Discussion}

In this EEG study, we used a tight controlled within-subjects design to systematically compare 9-and-12-month-old infants' distinct motor and visual processes to motorically familiar and unfamiliar actions using alpha power in the 6-9 Hz frequency range. More specifically, we investigated whether there are differences in central and occipital alpha during observation of motorically familiar versus unfamiliar actions. We extended our analyses by exploring associations of behavioral competence and mu power, and by investigating functional connectivity in the 6-9 Hz frequency range.

We pursued several methodological approaches, including using baselines that carefully matched the visual aspects of the experimental window, to more clearly assess distinct actionspecific motor process and visual attentional processes in response to familiar versus novel actions. We found that both mu suppression and occipital alpha suppression were evident during observations of both motorically familiar and unfamiliar actions, though they were distinct in the pattern they exhibited. Mu suppression was present during action anticipation (before movements occurred) and throughout the movement period, while occipital alpha was present during the onset of movement and not during action anticipation. This suggests that mu suppression is independent of an attentional response to the visual aspects of the stimulus. More importantly, mu suppression was stronger for familiar than for novel actions, whereas the level of occipital alpha suppression did not vary as a function of motor familiarity. While this replicates Southgate \& Begus (2013) and Virji-Babul et al. (2012) by showing that mu suppression is evident during observation of familiar and novel action, these findings also differ from previous ones in that mu suppression is sensitive to motoric familiarity. Virji-Babul et al. (2012) did not report occipital alpha power and thus it is unclear to what extent alpha effects were driven by motor or visual areas. In addition to not finding a difference between central and occipital alpha as a function of motor familiarity, Southgate \& Begus (2013) found an increase in occipital alpha from baseline. This might reflect potential visual effects related to the demand of the task beyond motor processes that may have affected their findings, but this possibility remains unexplored. 
Consistent with prior research relating expertise in action and neural motor activity during observation of familiar action in adults and infants (Calvo-Merino et al., 2006; Cross et al., 2006; Cannon et al., 2014, 2016; Orgs et al., 2008), we found that mu suppression was specifically associated with infants' own competence in actions. We found a correlation between 9-monthold infants' grasp latency and the level of mu power during observation of grasping actions. For occipital alpha no such relation was evident. It should be noted that the lack of evidence for an association between mu power and grasp competence in the 12-month-old group is not necessarily an indication of no relation, but could be due to little variability within the 12month-old grasp competence. Indeed, as infants gain more experience with an action, they produce more stable and accurate movements (Thelen et al., 1996; von Hofsten, 2004). Thus, we provide supporting evidence that motor competence is associated with mu power for familiar action, that is not found with occipital alpha.

Also, we found no evidence for such relation between mu suppression during observation of novel actions and infants' competence in novel actions. While, in general the interpretation on the lack of evidence for a relation should be interpreted with extreme caution, it is in this case not surprising and was expected given that the novel action is outside of the infants' motor repertoire at this age. It is an open question whether we would observe a change in this relation as infants gain experience and learn to produce means-end actions. Future work should directly address this possibility.

Together, while our results regarding mu power show that mu suppression is exhibited for both familiar and novel actions, we also observe a unique pattern for motoric familiarity in the way that is reflected in mu suppression. The level mu suppression is modulated by motoric familiarity, and mu suppression during observation of familiar action specifically correlates with infants' own actions. Nevertheless, it is difficult to reconcile the puzzle on the underlying neural correlates of perception of motorically familiar action by relying on mu power alone.

Exploring functional connectivity between networks, rather than isolated regional neural markers, may be a better indicator regarding the underlying mechanism during perception of 
motorically familiar action. Critically, many infant EEG studies report activation of mu and occipital alpha during observation of actions (Cannon et al., 2016; Yoo et al., 2015; Filippi et al., 2016; de Klerk et al., 2016); however, only few have directly explored whether activity from central and occipital alpha are functionally related during action observation (Debnath et al., 2019). When we extend our analyses beyond mu power to the functional connectivity of motor and visual processes, we find a clearer role of motor familiarity on neural processes of action perception. During observation of familiar grasping actions, central-occipital phase coherence in the $6-9 \mathrm{~Hz}$ frequency range was higher than phase coherence between areas with shorter distance like central-parietal and central-frontal electrode pairs across age. In other words, central and occipital areas were functionally more connected compared to their connections with other brain regions. Debnath et al. (2019) suggested that higher phase synchronization between central and occipital regions indicates that while activity in motor and visual regions reflect distinct processes, they are functionally linked. We did not find evidence for such a specific connectivity pattern during observation of the novel tool-use action. Thus, we introduce the possibility that neural correlates of perceiving motorically familiar versus unfamiliar action may be reflected in integrated activity of a neural network.

Understanding the dynamic of interconnected networks in the brain can provide insights on functional capabilities infancy (Gao et al., 2017). As such, the application of functional connectivity in this study sheds light on the potential possibility of inter-relation between motor experience and motor-visual connectivity. That is, the functional connectivity between motor and visual regions during observation of familiar grasping action may be a product of active experience in grasping and observing one's grasp. Infants start grasping for objects at 3-4 months and become proficient in grasping around 8 to 9 months of age (von Hofsten \& FazelZandy, 1984). Actions such as grasping, allow observation of direct physical changes. For instance, when one produces a grasping action, the effect of grasping is clearly visible in that the object moves closer and looks bigger (Woodward, 2005). Therefore, accumulated experiences with producing grasps and observing their effects could have built on stronger connections between motor and visual regions, thereby functionally playing a role in perception of others' grasps. In fact, Pineda (2005) hypothesized that coupling between 
different sources of activity could be responsible for the relation between perception and action, and researchers already have emphasized the potential functional significance of motorvisual coactivation (Fox et al., 2016), specifically in the developmental population (Bowman et al., 2017). This also aligns well with the theory on bidirectional action-effect associations which stresses the link between motor system activation and visual processing during action perception (Paulus, 2012). More work is needed, yet our findings highlight the potential significance of exploring the dynamics of interconnected networks in the infant brain, which may better illuminate the underlying processes of action perception.

Future direction

An open question is whether and to what extent new motor experience with a novel action modulates neural activity in infancy. At the behavioral level, extensive literature has demonstrated that active experience on a novel action alters infants' processing of others' actions (Gerson \& Woodward, 2014; Gerson, Mahajan et al., 2015; Libertus \& Needham, 2010; Sommerville et al., 2005, 2008). For instance, Sommerville et al. (2008) trained 10-month-old infants on using a cane to retrieve an out-of-reach toy. They found that having this active experience changed infants' goal understanding of another person's cane-use action. EEG studies have found consistent results on the role of active experience when processing others actions as well (Bakker et al., 2016; Gerson, Bekkering et al., 2015; Paulus et al., 2012). Our study contributes to the literature by showing that active experience with a familiar action is associated with stronger mu suppression and with neural communication between motor and visual areas during observation. However, with the current analysis, we cannot draw any conclusions about whether mu power or functional connectivity is modulated by learning a novel skill. It will be important to corroborate whether active experience or training of novel action subsequently generates stronger mu power to trained action and whether training induces changes in functional connectivity between motor and visual processes.

Another crucial direction would be to build upon our understanding of the dynamic communication between regions during action perception in infancy. Our study expanded on 
the approach to explore connectivity between regions, and it revealed critical implications on action perception. However, it is restricted to regional connectivity and does not consider interactions across large scale neural networks. Computational approaches and methodological innovation in this regard would be important to depict a comprehensive picture of connections across the whole brain during action perception, and further elucidate how the network of communication between areas changes as a function of experience.

Lastly, in order to accurately depict whether and how active motoric experience affects generating efficient connectivity routes during action perception, research has yet to gain understanding on how motor skill acquisition changes neural processing, in particular structural and functional connectivity patterns. Surprisingly little is known and documented about the links between changes in early motor development and neural processes associated with production of action. Only a few studies document changes in EEG power or coherence associated with changes in motor skill in infancy (resting-state EEG-Bell \& Fox, 1996; Corbetta et al., 2014; Xiao et al., 2018; during action execution- Nishiyori et al., 2016; 2021). This is a critical gap in the literature that necessitates attention for better understanding of the relation and coordination between infants' motor control and changes in neural activity. Importantly, if changes in power or coherence coincide with changes in motor experience or ability, a holistic interpretation can be made regarding the role of motor experience on neural reorganization and the mechanism involved with the interplay between action perception and execution (Gonzalez et al., 2016).

\section{Conclusion}

Together, our findings provide fundamental evidence on the neural correlates and novel insights about motor-visual processes during infants' action perception of familiar and novel actions. Specifically, observation of familiar actions exhibited stronger motor-related response distinct from general visual processes, and was associated with specific functional connectivity between motor and visual areas compared to connections with other brain regions. This paves the way for future research to make use of methodological approaches to parse motor-specific 
Neural Correlates of Familiar and Unfamiliar Action in Infancy

neural activity while also to consider neural processing in a dynamic network. This further broadens the scope of infant EEG mu rhythm procedures and its interpretations.

\section{Funding}

This research was supported by Eunice Kennedy Shriver National Institute of Child Health and Human Development Grant (P01- HD064653) and National Science Foundation (NSF 1628300) awarded to A. Woodward and N. Fox.

\section{Acknowledgements}

We thank the families who participated in our study. We also wish to acknowledge the contributions of our lab manager Riley Abeles and research Rebecca Wixted and Christina Hartman for their help with testing and video coding. 
Neural Correlates of Familiar and Unfamiliar Action in Infancy

\section{References}

Ambrosini, E., Reddy, V., de Looper, A., Costantini, M., Lopez, B., \& Sinigaglia, C. (2013). Looking Ahead: Anticipatory Gaze and Motor Ability in Infancy. PLoS ONE, 8(7), e67916. https://doi.org/10.1371/journal.pone.0067916

Bakker, M., Sommerville, J. A., \& Gredebäck, G. (2016). Enhanced neural processing of goaldirected actions after active training in 4-month-old infants. Journal of cognitive neuroscience, 28(3), 472-482. https://doi.org/10.1162/jocn_a_00909

Bell, M. A., \& Fox, N. A. (1994). Brain development over the first year of life: Relations between electroencephalographic frequency and coherence and cognitive and affective behaviors. In G. Dawson \& K. W. Fischer (Eds.), Human behavior and the developing brain (pp. 314-345). The Guilford Press.

Bertenthal, B. I., \& Clifton, R. K. (1998). Perception and action. In W. Damon (Ed.), Handbook of child psychology: Vol. 2. Cognition, perception, and language (p. 51-102). John Wiley \& Sons Inc.

Bowman, L. C., Bakermans-Kranenburg, M. J., Yoo, K. H., Cannon, E. N., Vanderwert, R. E., Ferrari, P. F., van IJzendoorn, M. H., \& Fox, N. A. (2017). The mu-rhythm can mirror: Insights from experimental design, and looking past the controversy. Cortex, 96, 121125. https://doi.org/10.1016/j.cortex.2017.03.025

Calvo-Merino, B., Grèzes, J., Glaser, D. E., Passingham, R. E., \& Haggard, P. (2006). Seeing or doing? Influence of visual and motor familiarity in action observation. Current biology, 16(19), 1905-1910.

Cannon, E. N., Simpson, E. A., Fox, N. A., Vanderwert, R. E., Woodward, A. L., \& Ferrari, P. F. (2016). Relations between infants' emerging reach-grasp competence and event-related desynchronization in EEG. Developmental Science, 19(1), 50-62.

https://doi.org/10.1111/desc.12295 
Neural Correlates of Familiar and Unfamiliar Action in Infancy

Cannon, E. N., Yoo, K. H., Vanderwert, R. E., Ferrari, P. F., Woodward, A. L., \& Fox, N. A. (2014). Action experience, more than observation, influences mu rhythm desynchronization. Plos one, 9(3), e92002. https://doi.org/10.1371/journal.pone.0092002

Cochin, S., Barthelemy, C., Roux, S., \& Martineau, J. (1999). Observation and execution of movement: Similarities demonstrated by quantified electroencephalography: qEEG of observation and execution of movement. European Journal of Neuroscience, 11(5), 1839-1842. https://doi.org/10.1046/j.1460-9568.1999.00598.x

Cohen, M.X., 2014. Analyzing Neural Time Series Data: Theory and Practice. MIT Press.

Corbetta, D., Friedman, D. R., \& Bell, M. A. (2014). Brain reorganization as a function of walking experience in 12-month-old infants: implications for the development of manual laterality. Frontiers in Psychology, 5, 245. https://doi.org/10.3389/fpsyg.2014.00245

Cross, E. S., Hamilton, A. F. D. C., \& Grafton, S. T. (2006). Building a motor simulation de novo: observation of dance by dancers. Neuroimage, 31(3), 1257-1267.

Cuevas, K., Cannon, E. N., Yoo, K., \& Fox, N. A. (2014). The infant EEG mu rhythm: Methodological considerations and best practices. Developmental Review, 34(1), 26-43. https://doi.org/10.1016/j.dr.2013.12.001

Datavyu Team (2014). Datavyu: A Video Coding Tool. Databrary Project, New York University. URL http://datavyu.org.

de Klerk, C. C. J. M., Southgate, V., \& Csibra, G. (2016). Predictive action tracking without motor experience in 8-month-old infants. Brain and Cognition, 109, 131-139. https://doi.org/10.1016/j.bandc.2016.09.010

Delorme, A., \& Makeig, S. (2004). EEGLAB: an open source toolbox for analysis of single-trial EEG dynamics including independent component analysis. Journal of neuroscience methods, 134(1), 9-21. https://doi.org/10.1016/j.jneumeth.2003.10.009 
Neural Correlates of Familiar and Unfamiliar Action in Infancy

Debnath, R., Buzzell, G. A., Morales, S., Bowers, M. E., Leach, S. C., \& Fox, N. A. (2020). The Maryland analysis of developmental EEG (MADE) pipeline. Psychophysiology, 57(6). https://doi.org/10.1111/psyp.13580

Debnath, R., Salo, V. C., Buzzell, G. A., Yoo, K. H., \& Fox, N. A. (2019). Mu rhythm desynchronization is specific to action execution and observation: Evidence from timefrequency and connectivity analysis. Neurolmage, 184, 496-507. https://doi.org/10.1016/j.neuroimage.2018.09.053

Filippi, C. A., Cannon, E. N., Fox, N. A., Thorpe, S. G., Ferrari, P. F., \& Woodward, A. L. (2016). Motor system activation predicts goal imitation in 7-month-old infants. Psychological science, $27(5), 675-684$.

Filippi, C. A., \& Woodward, A. L. (2016). Action Experience Changes Attention to Kinematic Cues. Frontiers in Psychology, 7. https://doi.org/10.3389/fpsyg.2016.00019

Fox, N. A., Bakermans-Kranenburg, M. J., Yoo, K. H., Bowman, L. C., Cannon, E. N., Vanderwert, R. E., Ferrari, P. F., \& van IJzendoorn, M. H. (2016). Assessing human mirror activity with EEG mu rhythm: A meta-analysis. Psychological Bulletin, 142(3), 291-313. https://doi.org/10.1037/bul0000031

Gallese, V., Fadiga, L., Fogassi, L., \& Rizzolatti, G. (1996). Action recognition in the premotor cortex. Brain, 119(2), 593-609. https://doi.org/10.1093/brain/119.2.593

Gao, W., Lin, W., Grewen, K., \& Gilmore, J. H. (2017). Functional connectivity of the infant human brain: Plastic and modifiable. Neuroscientist, 23(2), 169-184. https://doi.org/10.1177/1073858416635986

Gerson, S. A., Bekkering, H., \& Hunnius, S. (2015). Short-term Motor Training, but Not Observational Training, Alters Neurocognitive Mechanisms of Action Processing in Infancy. Journal of Cognitive Neuroscience, 27(6), 1207-1214. https://doi.org/10.1162/jocn_a_00774 
Neural Correlates of Familiar and Unfamiliar Action in Infancy

Gerson, S. A., Mahajan, N., Sommerville, J. A., Matz, L., \& Woodward, A. L. (2015). Shifting goals: Effects of active and observational experience on infants' understanding of higher order goals. Frontiers in Psychology, 6, 310. https://doi.org/10.3389/fpsyg.2015.00310

Gerson, S. A., \& Woodward, A. L. (2014). The joint role of trained, untrained, and observed actions at the origins of goal recognition. Infant Behavior and Development, 37(1), 94104. https://doi.org/10.1016/j.infbeh.2013.12.013

Gonzalez, S. L., Reeb-Sutherland, B. C., \& Nelson, E. L. (2016). Quantifying Motor Experience in the Infant Brain: EEG Power, Coherence, and Mu Desynchronization. Frontiers in Psychology, 7, 216. https://doi.org/10.3389/fpsyg.2016.00216

Hari, R., Salmelin, R., Mäkelä, J. P., Salenius, S., \& Helle, M. (1997). Magnetoencephalographic cortical rhythms. International Journal of Psychophysiology, 26, 51- 62. http://dx.doi.org/10.1016/S0167- 8760(97)00755-1

Hobson, H. M., \& Bishop, D. V. M. (2016). Mu suppression - A good measure of the human mirror neuron system? Cortex, 82, 290-310. https://doi.org/10.1016/j.cortex.2016.03.019

Hunnius, S., \& Bekkering, H. (2014). What are you doing? How active and observational experience shape infants' action understanding. Philosophical Transactions of the Royal Society B: Biological Sciences, 369(1644), 20130490. https://doi.org/10.1098/rstb.2013.0490

Hwang, H. G., Debnath, R., Meyer, M., Salo, V. C., Fox, N. A., \& Woodward, A. (2021). Neighborhood racial demographics predict infants' neural responses to people of different races. Developmental science, 24(4), e13070.

Johnson, M. H. (2000). Functional brain development in infants: Elements of an interactive specialization framework. Child development, 71(1), 75-81. https://doi.org/10.1111/1467-8624.00120 
Neural Correlates of Familiar and Unfamiliar Action in Infancy

Kanakogi, Y., \& Itakura, S. (2011). Developmental correspondence between action prediction and motor ability in early infancy. Nature Communications, 2(1), 341. https://doi.org/10.1038/ncomms1342

Kayser, J., \& Tenke, C. E. (2006). Issues and considerations for using the scalp surface Laplacian in EEG/ERP research: A tutorial review. International Journal of Psychophysiology, 97(3), 189-209. https://doi.org/10.1016/j.ijpsycho.2015.04.012

Keen, R. (2011). The development of problem solving in young children: A critical cognitive skill. Annual review of psychology, 62, 1-21. https://doi.org/10.1146/annurev.psych.031809.130730

Krogh-Jespersen, S., Henderson, A. M. E., \& Woodward, A. L. (2020). Let's get it together: Infants generate visual predictions based on collaborative goals. Infant Behavior and Development, 59, 101446. https://doi.org/10.1016/j.infbeh.2020.101446

Krogh-Jespersen, S., \& Woodward, A. L. (2018). Reaching the goal: Active experience facilitates 8-month-old infants' prospective analysis of goal-based actions. Journal of Experimental Child Psychology, 171, 31-45. https://doi.org/10.1016/j.jecp.2018.01.014

Lepage, J.-F., \& Théoret, H. (2006). EEG evidence for the presence of an action observationexecution matching system in children. European Journal of Neuroscience, 23(9), 25052510. https://doi.org/10.1111/j.1460-9568.2006.04769.x

Libertus, K., \& Needham, A. (2010). Teach to reach: The effects of active vs. passive reaching experiences on action and perception. Vision Research, 50(24), 2750-2757. https://doi.org/10.1016/j.visres.2010.09.001

Marshall, P. J., \& Meltzoff, A. N. (2014). Neural mirroring mechanisms and imitation in human infants. Philosophical Transactions of the Royal Society B: Biological Sciences, 369(1644), 20130620. https://doi.org/10.1098/rstb.2013.0620

Marshall, P. J., Young, T., \& Meltzoff, A. N. (2011). Neural correlates of action observation and execution in 14-month-old infants: An event-related EEG desynchronization study. 
Neural Correlates of Familiar and Unfamiliar Action in Infancy

Developmental science, 14(3), 474-480. https://doi.org/10.1111/j.14677687.2010.00991.x

Meyer, M., Chung, H., Debnath, R., Fox, N., \& Woodward, A. L. (2022). Social context shapes neural processing of others' actions in 9-month-old infants. Journal of Experimental Child Psychology, 213, 105260. https://doi.org/10.1016/j.jecp.2021.105260

Mognon, A., Jovicich, J., Bruzzone, L., Buiatti, M., 2011. ADJUST: an automatic EEG artifact detector based on the joint use of spatial and temporal features. Psychophysiology, 48, 229-240. https://doi.org/10.1111/j.1469-8986.2010.01061.x

Muthukumaraswamy, S. D., \& Johnson, B. W. (2004). Changes in rolandic mu rhythm during observation of a precision grip. Psychophysiology, 41(1), 152-156. https://doi.org/10.1046/j.1469-8986.2003.00129.x

Nishiyori, R., Bisconti, S., Meehan, S. K., \& Ulrich, B. D. (2016). Developmental changes in motor cortex activity as infants develop functional motor skills. Developmental Psychobiology, 58(6), 773-783. https://doi.org/10.1002/dev.21418

Nishiyori, R., Harris, M. K., Baur, K., \& Meehan, S. K. (2021). Changes in cortical hemodynamics with the emergence of skilled motor ability in infants: An fNIRS study. Brain research, 1772, 147666. https://doi.org/10.1016/j.brainres.2021.147666

Nolan, H., Whelan, R., \& Reilly, R. B. (2010). FASTER: fully automated statistical thresholding for EEG artifact rejection. Journal of Neuroscience Methods, 192(1), 152-162.

Nyström, P., Ljunghammar, T., Rosander, K., \& von Hofsten, C. (2011). Using mu rhythm desynchronization to measure mirror neuron activity in infants. Developmental science, 14(2), 327-335. https://doi.org/10.1111/j.1467-7687.2010.00979.x

Orgs, G., Dombrowski, J.-H., Heil, M., \& Jansen-Osmann, P. (2008). Expertise in dance modulates alphabeta event-related desynchronization during action observation. European Journal of Neuroscience, 27(12), 3380-3384. https://doi.org/10.1111/j.14609568.2008.06271.x 
Neural Correlates of Familiar and Unfamiliar Action in Infancy

Paulus, M. (2012). Action mirroring and action understanding: An ideomotor and attentional account. Psychological Research, 76(6), 760-767. https://doi.org/10.1007/s00426-0110385-9

Paulus, M., Hunnius, S., van Elk, M., \& Bekkering, H. (2012). How learning to shake a rattle affects 8-month-old infants' perception of the rattle's sound: Electrophysiological evidence for action-effect binding in infancy. Developmental Cognitive Neuroscience, 2(1), 90-96. https://doi.org/10.1016/j.dcn.2011.05.006

Perry, A., \& Bentin, S. (2010). Does focusing on hand-grasping intentions modulate electroencephalogram $\mu$ and $\alpha$ suppressions? NeuroReport, 21(16), 1050e1054.

Pineda, J. A. (2005). The functional significance of mu rhythms: Translating "seeing" and "hearing" into "doing." Brain Research Reviews, 50(1), 57-68. https://doi.org/10.1016/j.brainresrev.2005.04.005

Saby, J. N., Meltzoff, A. N., \& Marshall, P. J. (2013). Infants' somatotopic neural responses to seeing human actions: I've got you under my skin. PLoS One, 8(10), e77905. https://doi.org/10.1371/journal.pone.0077905

Schneider, W., Eschman, A., \& Zuccolotto, A. (2012). E-Prime User's Guide. Pittsburgh: Psychology Software Tools, Inc.

Sommerville, J. A., Hildebrand, E. A., \& Crane, C. C. (2008). Experience matters: The impact of doing versus watching on infants' subsequent perception of tool-use events. Developmental Psychology, 44(5), 1249-1256. https://doi.org/10.1037/a0012296

Sommerville, J. A., Woodward, A. L., \& Needham, A. (2005). Action experience alters 3-monthold infants' perception of others' actions. Cognition, 96(1), B1-B11. https://doi.org/10.1016/j.cognition.2004.07.004

Southgate, V., \& Begus, K. (2013). Motor Activation During the Prediction of Nonexecutable Actions in Infants. Psychological Science, 24(6), 828-835. https://doi.org/10.1177/0956797612459766 
Neural Correlates of Familiar and Unfamiliar Action in Infancy

Southgate, V., Johnson, M. H., Osborne, T., \& Csibra, G. (2009). Predictive motor activation during action observation in human infants. Biology Letters, 5(6), 769-772. https://doi.org/10.1098/rsbl.2009.0474

Tangwiriyasakul, C., Verhagen, R., van Putten, M. J. A. M., \& Rutten, W. L. C. (2013). Importance of baseline in event- related desynchronization during a combination task of motor imagery and motor observation. Journal of Neural Engineering, 10(2), 026009. http://dx.doi.org/10.1088/1741- 2560/10/2/026009.

Thelen, E., Corbetta, D., \& Spencer, J. P. (1996). Development of reaching during the first year: role of movement speed. Journal of experimental psychology: human perception and performance, 22(5), 1059.

Toro, C., Deuschl, G., Thatcher, R., Sato, S., Kufta, C., \& Hallett, M. (1994). Event-related desynchronization and movement-related cortical potentials on the ECoG and EEG. Electroencephalography and Clinical Neurophysiology, 93, 380 -389. http://dx.doi.org/10.1016/0168- 5597(94)90126-0

van Elk, M., van Schie, H. T., Hunnius, S., Vesper, C., \& Bekkering, H. (2008). You'Il never crawl alone: Neurophysiological evidence for experience-dependent motor resonance in infancy. Neurolmage, 43(4), 808-814. https://doi.org/10.1016/j.neuroimage.2008.07.057

Virji-Babul, N., Rose, A., Moiseeva, N., \& Makan, N. (2012). Neural correlates of action understanding in infants: Influence of motor experience. Brain and Behavior, 2(3), 237242. https://doi.org/10.1002/brb3.50

von Hofsten, C. (2004). An action perspective on motor development. Trends in cognitive sciences, 8(6), 266-272.

von Hofsten, C., \& Fazel-Zandy, S. (1984). Development of visually guided hand orientation in reaching. Journal of Experimental Child Psychology, 38(2), 208-219.

https://doi.org/10.1016/0022-0965(84)90122-X 
Neural Correlates of Familiar and Unfamiliar Action in Infancy

Widmann, A., Schröger, E., \& Maess, B. (2015). Digital filter design for electrophysiological data-a practical approach. Journal of neuroscience methods, 250, 34-46. https://doi.org/10.1016/j.jneumeth.2014.08.002

Woodward, A. L. (2005). Infants' Understanding of the Actions. Joint attention: Communication and other minds: Issues in philosophy and psychology, 110.

Woodward, A. L., \& Gerson, S. A. (2014). Mirroring and the development of action understanding. Philosophical Transactions of the Royal Society B: Biological Sciences, 369(1644), 20130181. https://doi.org/10.1098/rstb.2013.0181

Xiao, R., Shida-Tokeshi, J., Vanderbilt, D. L., \& Smith, B. A. (2018). Electroencephalography power and coherence changes with age and motor skill development across the first half year of life. PLoS One, 13(1), e0190276. https://doi.org/10.1371/journal.pone.0190276

Yoo, K. H., Cannon, E. N., Thorpe, S. G., \& Fox, N. A. (2016). Desynchronization in EEG during perception of means-end actions and relations with infants' grasping skill. British Journal of Developmental Psychology, 34(1), 24-37. https://doi.org/10.1111/bjdp.12115 